\title{
EXTRATOS AQUOSOS DE PLANTAS E O COMPORTAMENTO DO ÁCARO VERDE DA MANDIOCA
}

\author{
Manoel Eneas de Carvalho Gonçalves ${ }^{1}$; José Vargas de Oliveira ${ }^{1,2 *}$; Reginaldo Barros ${ }^{1}$; \\ Marcileyne Pessôa Leite de Lima ${ }^{1}$ \\ ${ }^{1}$ Depto. de Agronomia - Fitossanidade/UFRPE, Av. Dom. Manoel de Medeiros s/n, Dois Irmãos - CEP: 52171-900 - \\ Recife, $P E$. \\ ${ }^{2}$ Bolsista CNPq. \\ *Autor correspondente <vargasoliveira@uol.com.br >
}

RESUMO: Extratos de plantas são uma alternativa promissora no controle de ácaros fitófagos. Neste trabalho estudou-se a bioatividade de extratos aquosos de nim, Azadirachta indica A. Juss. e cravo-da-índia, Syzigium aromaticum L., na biologia do ácaro verde da mandioca, Mononychellus tanajoa (Bondar). Larvas recémeclodidas foram individualizadas em discos de folhas de mandioca previamente imersos nos extratos e em água destilada, e mantidas nestas condições até atingir a fase adulta. Após a emergência, as fêmeas foram individualizadas em discos não tratados e acasaladas com machos coletados da criação estoque. As avaliações foram efetuadas a cada $12 \mathrm{~h}$. As duas maiores concentrações de nim (1,0 e $0,75 \mathrm{~g}$ do pó de sementes de nim $100 \mathrm{~mL}^{-1}$ de água) deram os melhores resultados, causando 72,5 e $37,5 \%$ de mortalidade larval, respectivamente, e até $16 \%$ para os estágios seguintes. A mortalidade em todo estágio imaturo foi de 84,6 e $55 \%$, respectivamente. A duração da fase deutocrisálida e teliocrisálida foi levemente afetada, porém significativamente maior para o nim a 1\%. Os extratos não afetaram a fecundidade, a qual variou de 6,5 a 8,6 ovos/fêmea/dia. Embora os extratos usados não tenham afetado o período de incubação, nim a $1 \%$ reduziu significativamente a viabilidade dos ovos.

Palavras-chave: Mononychellus tanajoa, Tetranychidae, acari, planta inseticida, praga

\section{AQUEOUS PLANT EXTRACTS AND THE BEHAVIOR OF THE CASSAVA GREEN MITE}

\begin{abstract}
The use of plant extracts is a promising alternative for the control of phytophagous mites. This study was carried out to test the bio-activity of neem, Azadirachta indica A. Juss. and clove, Syzigium aromaticum L., aqueous extracts on the behaviour of the cassava green mite, Mononychellus tanajoa (Bondar). Recently hatched larvae were individualized onto cassava leaf discs previously immersed in different concentrations of the extract and in distilled water, and maintained at these conditions until reaching maturity. The females were individualized onto untreated leaf discs right after emergency and mated with males from the rearing stock. The evaluations were taken each $12 \mathrm{~h}$. The two highest neem concentrations ( 1.0 and $0.75 \mathrm{~g}$ of ground neem seeds $100 \mathrm{~mL}^{-1}$ water) gave the best results, causing 72.5 and $37.5 \%$ of larval mortality, respectively. Mortality of other stages was up $16 \%$. The mortality of the combined immature stages was 84.6 and $55 \%$, at neem concentrations of 1.0 and $0.75 \%$, respectively. The duration of the deutochrysalis and teleiochrysalis stages was slightly but significantly longer for neem at $1.0 \%$ probability. The plant extracts did not affect female fecundity, which ranged from 6.5 to 8.6 eggs/female/day. Although the extracts used did not affect the incubation period, neem extract significantly reduced egg viability at $1 \%$ probability.

Key words: Mononychellus tanajoa, Tetranychidae, acari, insecticide plant, pest
\end{abstract}

\section{INTRODUÇÃO}

O ácaro verde, Mononychellus tanajoa (Bondar), é considerado praga de grande importância econômica para a cultura da mandioca na América do Sul e em países do continente Africano (Yaninek et al., 1989). Em condições de temperatura elevada e umidade relativa baixa, desenvolvem altas infestações, iniciando-se pela parte superior das plantas, atacando as gemas e folhas novas, as quais tornam-se cloróticas e deformadas, com pontuações amareladas (Samways, 1979; Bellotti et al., 1983). Em Pernambuco, Brasil, este ácaro tem causado perdas de até $51 \%$ na produção de raízes. Na África, as perdas têm atingido até 80\% (Byrne et al., 1983; Veiga, 1985; Yaninek \& Herren, 1988).
As táticas de controle, como cultivares resistentes, práticas culturais e controle biológico com liberações de ácaros predadores, têm sido utilizadas na redução de infestações do ácaro verde, em diferentes regiões produtoras (Doreste et al., 1979; Byrne et al., 1982; Bellotti et al., 1983; Moraes, 1991; Noronha et al.,1995). Veiga (1985) constatou a eficácia de produtos químicos no controle do ácaro verde no Estado de Pernambuco. No entanto, o controle químico é considerado antieconômico, pelo longo ciclo da cultura, o baixo retorno econômico e os recursos limitados dos produtores (Bellotti et al., 1999). Além disso McMurtry et al. (1970) mencionaram a possibilidade de efeitos adversos decorrentes do uso desses produtos, como a redução populacional de 
inimigos naturais e o desenvolvimento de linhagens de ácaros resistentes.

Como uma opção de menor custo, os extratos vegetais, são atualmente alvo de pesquisas como método alternativo de controle de ácaros fítófagos. Os extratos de nim Azadirachta indica A. Juss. e formulações à base de azadiractina, um composto tetranortriterpenóide com ação inseticida e acaricida presente nas sementes de nim (Schmutterer, 1990), têm apresentado ação sobre os ácaros vermelho Tetranychus cinnabarinus (Boisd.) e rajado Tetranychus urticae Koch, causando inibição da oviposição, redução da viabilidade de ovos e mortalidade de formas imaturas e adultos (Mansour \& Ascher, 1983; Mansour et al., 1987; Dimetry et al., 1993; Mansour et al., 1993; Sundaram et al., 1995; Mansour et al., 1997; Miller \& Uetz, 1998). No levantamento bibliográfico efetuado somente foi encontrado uma referência sobre o uso de extratos vegetais no controle do ácaro verde, na qual extratos aquosos de nim e cravo-da-índia, Syzigium aromaticum L., nas concentrações 5 e $10 \%$ provocaram mortalidade de fêmeas entre 85 e $100 \%$ (Gonçalves et al., 1999). Adicionalmente, os compostos bioativos do nim têm apresentado seletividade para organismos não alvo, como os inimigos naturais de ácaros (Mansour et al., 1986; Mansour et al., 1987; Spollen \& Isman, 1996). Assim, devido à ausência de produtos formulados à base de nim no mercado brasileiro, e buscando desenvolver tecnologias para utilização de extratos vegetais, com baixo custo para o produtor, este trabalho teve como objetivo estudar a bioatividade de extratos aquosos de plantas de nim e cravo-da-índia sobre a biologia do ácaro verde da mandioca.

\section{MATERIAL E MÉTODOS}

O trabalho foi realizado no laboratório de Entomologia Agrícola da Universidade Federal Rural de Pernambuco (UFRPE), a $27 \pm 0,5^{\circ} \mathrm{C}$, umidade relativa de $69 \pm 4 \%$ e fotofase de $12 \mathrm{~h}$.

Criação estoque de $\boldsymbol{M}$. tanajoa. Folhas de mandioca do cultivar Santo Estevão infestadas pelo ácaro verde foram coletadas no campus da UFRPE, e levadas ao laboratório. Estas foram dispostas em papel de filtro sobre uma camada de algodão umedecido, no interior de bandejas de isopor com dimensões de $20 \times 15 \times 2$ $\mathrm{cm}$. As fêmeas foram transferidas com pincel de pelo fino para discos de folhas de $3,5 \mathrm{~cm}$ de diâmetro do mesmo cultivar.

Obtenção do material vegetal e preparo dos extratos. As sementes de nim foram coletadas de plantas com cerca de três anos de idade em bosques da Embrapa Semi-Árido, em Petrolina, PE, o cravo-da-índia foi adquirido em supermercado na cidade de Recife, PE, em embalagens herméticas com validade de 12 meses. As amêndoas obtidas de sementes de nim colhidas 46 dias antes e botões de cravo-da-índia foram triturados, separadamente, em moinho de facas. Os extratos foram preparados misturando-se $0,5,0,75,1,0 \mathrm{~g}$ de amêndoas de sementes de nim ou 5,0 g de flores de cravo-da-índia, separadamente, em recipientes de vidro com $100 \mathrm{~mL}$ de água destilada. As misturas foram deixadas em repouso durante $24 \mathrm{~h}$ para extração das substâncias secundárias hidrossolúveis, sendo posteriormente filtradas com tecido tipo voal. As concentrações dos extratos são referidas como $0,5,0,75$ e 1,0\% de nim e 5,0\% de cravo-da-Índia, respectivamente.

Aplicação dos extratos. Discos de folhas do cultivar Santo Estevão foram imersos nos extratos por 10 segundos, agitados levemente e colocados para secar em condições ambiente durante 30 minutos. Em seguida, estes foram dispostos em papel de filtro sobre uma camada de algodão umedecido, mantidos no interior de bandejas de isopor. Discos imersos da mesma forma em água destilada foram usados como testemunha.

Avaliação dos extratos. Imediatamente após o processo anteriormente citado, larvas recém - eclodidas de $M$. tanajoa foram individualizadas em discos tratados e testemunha, e mantidas nestas condições até o final do estágio imaturo. A cada dois dias fez-se a substituição dos discos, por outros tratados com as mesmas concentrações, procedendo-se às avaliações em intervalos de 12 horas. Ao atingirem a fase adulta, as fêmeas foram individualizadas em discos não tratados e acasaladas com machos da criação estoque. $O$ efeito dos extratos foi avaliado através da observação da mortalidade e duração das fases imaturas, fecundidade e fertilidade de fêmeas. $O$ delineamento utilizado foi o inteiramente casualizado com cinco tratamentos e quatro repetições, sendo o tratamento composto por 10 indivíduos em cada repetição. Os resultados foram submetidos à análise de variância e as médias comparadas pelo teste de Tukey $(P \leq 0,05)$.

\section{RESULTADOS E DISCUSSÃO}

No estágio larval de $M$. tanajoa, os extratos de nim 1,0 e $0,75 \%$ causaram 72,5 e $37,5 \%$ de mortalidade, respectivamente (Figura 1). Nos estágios posteriores, observou-se o máximo de $16 \%$ de mortalidade. Nim 0,5\% causou reduzida mortalidade nos estágios de larva, protocrisálida e teliocrisálida, e o cravo-da-índia $5,0 \%$ causou somente $5 \%$ de mortalidade no estágio de teliocrisálida, não afetando os demais. Os extratos de nim 0,75 e $1,0 \%$ causaram mortalidade acumulada de 55 e $84,6 \%$ na fase de larva a teliocrisálida, diferindo de nim $0,5 \%$ e cravo-da-índia $5,0 \%$, com mortalidade de 11,1 e $5,4 \%$, 
respectivamente (Figura 2). Segundo Mansour \& Ascher (1983), os extratos aquosos de nim não foram eficazes, em relação aos extratos orgânicos clorofórmico, n-butanólico, acetônico, pentânico e metanólico, quanto à mortalidade dos estágios imaturos de $T$. cinnabarinus, apresentando $\mathrm{CL}_{50}$ de 0,$13 ; 0,13 ; 0,16 ; 0,2$ e $0,8 \%$, respectivamente. Indicaram assim que os compostos orgânicos (apolares) extraíram maior quantidade de substâncias secundárias, que a água (polar). A mortalidade causada pelos extratos de nim 0,75 e 1\%, no presente trabalho deveu-se provavelmente à ação de compostos secundários presentes nas sementes de nim. Segundo Rembold (1989) e Schmutterer (1990), o azadiractina é o principal composto bioativo quantitativamente isolado de sementes desta meliácea. A baixa eficácia alcançada pelo extrato de nim $0,5 \%$, pode ter sido devido a uma menor quantidade destes compostos em relação a nim 1,0 e $0,75 \%$. Miller \& Uetz (1998) avaliaram a ação de formulações comerciais em estágios imaturos e adulto do ácaro rajado, Tetranychus urticae Koch, constatando que Margosan- $\mathrm{O}$ ( $0,3 \%$ de azadiractina) pulverizado na dose de $4,7 \mathrm{~mL} \mathrm{~L}^{-1}$, em folhas de cravode-defunto, Tagetes patula L., causou $100 \%$ de mortalidade após três dias da aplicação. O cravo-daíndia 5,0\% causou mortalidade inexpressiva dos estágios imaturos de $M$. tanajoa, diferindo dos resultados de Gonçalves et al. (1999), que obtiveram $100 \%$ de mortalidade de fêmeas deste ácaro com o uso da técnica de imersão de lâminas. A baixa mortalidade causada pelos extratos de nim a 0,75 e $1,0 \%$, sobre os estágios pós-larvais, sugere que estes sejam mais tolerantes aos compostos bioativos nestas concentrações, do que o estágio larval. Outra
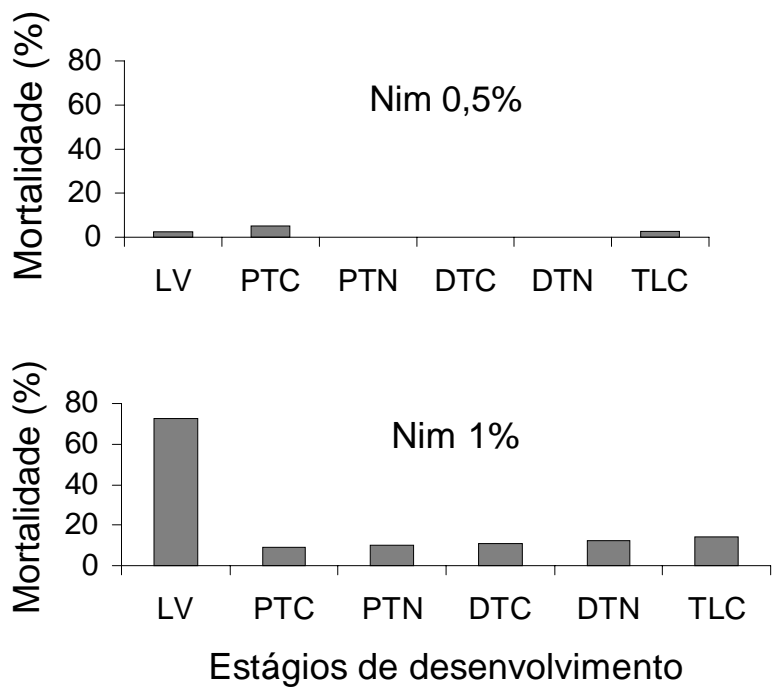

explicação para este fato seria que os extratos aquosos apresentaram baixo poder residual, porém não se tem informações sobre a persistência destes em condições de laboratório. Mansour \& Ascher, (1983) observaram que após sete dias, os extratos acetônico e pentânico de nim a $1 \%$ ainda causaram mortalidade para fêmeas de $T$. cinnabarinus.

No geral, os extratos não apresentaram grande influência na duração dos estágios imaturos ativos e quiescentes do ácaro verde, embora significativa para os estágios de deutocrisálida e teliocrisálida quando tratados com o nim a 1,0\% (TABELA 1). Não foram detectadas diferenças significativas entre os tratamentos em relação à fecundidade, que variou de 6,5 a 8,6 ovos/fêmea/dia (Figura 3). Mansour et al. (1997), avaliando o efeito residual da formulação Neemgard (4,5\% de azadiractina) $1 \%$ sobre fêmeas de T. cinnabarinus em feijoeiro, observaram redução de 96,4 e $85 \%$ no número de ovos/dia, em relação à testemunha, quando expostas a resíduos de 4 e 8 dias, respectivamente. A fecundidade do ácaro rajado T. urticae, em discos de folhas de framboesa tratados com as formulações comerciais Margosan-O $(0,3 \%$ de Azadiractina-AZA) e Neem azal-S $(0,35 \%$ de AZA) na concentração $0,4 \%$, foi reduzida em 83,3 e $92,6 \%$, respectivamente (Dimetry et al., 1993). No entanto, Mansour \& Ascher (1983) observaram a ineficácia de extratos aquosos de nim sobre a fecundidade de $T$. cinnabarinus em discos de folhas de feijoeiro, e a eficácia de extratos orgânicos da mesma planta. Os extratos metanólicos, etanólicos, acetônicos e pentânicos reduziram a fecundidade deste ácaro em $35,50,89$ e $97 \%$, na concentração de $0,5 \%$, e em 50 , 72, 94 e 94\%, respectivamente, na concentração de 1,0\% (Mansour et al., 1987). Neste estudo, esperava-

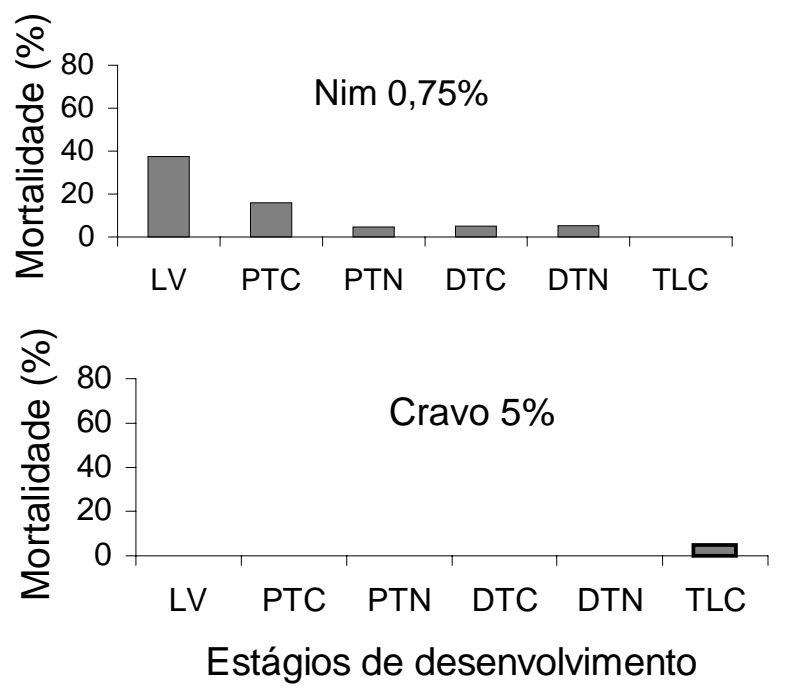

Figura 1 - Mortalidade de cada estágio imaturo do ácaro verde, M. tanajoa, em discos de folhas de mandioca tratados com extratos aquosos de nim, A. indica, e cravo-da-índia, S. aromaticum. (LV) larva; (PTC) protocrisálida; (PTN) protoninfa; (DTC) deutocrisálida; (DTN) deutoninfa; (TLC) teliocrisálida. Temp: $27 \pm 0,5^{\circ} \mathrm{C}, 69 \pm 4 \%$ de UR e fotofase de $12 \mathrm{~h}$. 
se que os extratos de nim consumidos na fase imatura pudessem afetar a reprodução das fêmeas adultas. Provavelmente, uma redução na postura e fertilidade poderia ocorrer se estas tivessem sido mantidas em discos tratados.

Os extratos não afetaram a duração do período de incubação, em relação à testemunha, com uma variação de 4,3 a 4,4 dias, considerando-se os ovos depositados no segundo dia de postura. Porém, o extrato de nim $1,0 \%$ reduziu em $14,8 \%$ a viabilidade dos ovos, não diferindo dos extratos de cravo-da-índia 5,0\%, nim 0,75 e $0,5 \%$ (Figura 4). A viabilidade dos ovos do ácaro rajado foi reduzida em 13,6 e $20,6 \%$ e o período de incubação foi aumentado de 1,05 e 0,95 dias, com as formulações comerciais Margosan-O $(0,3 \%$ de Azadiractina-AZA) e Neem azal-S $(0,35 \%$ de AZA) na concentração $0,4 \%$, respectivamente (Dimetry et al., 1993).

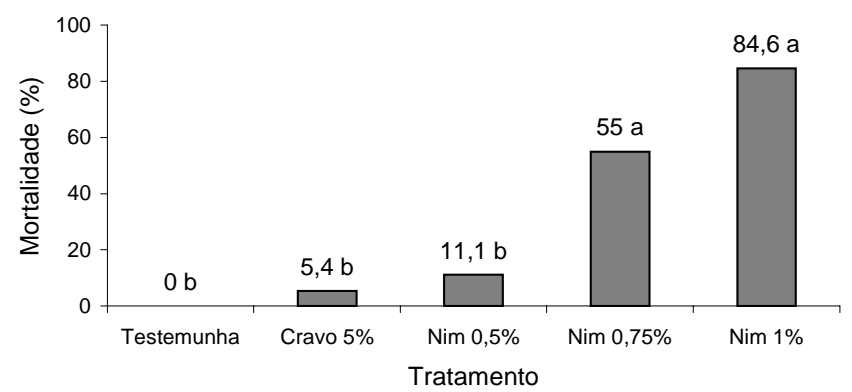

Figura 2 - Mortalidade acumulada da fase de larva a teliocrisálida do ácaro verde, M. tanajoa, em discos de folhas de mandioca tratados com extratos aquosos de nim, $A$. indica, e cravo-da-índia, S. aromaticum. Temp: $27 \pm 0,5^{\circ} \mathrm{C}$, $69 \pm 4 \%$ de UR e fotofase de $12 \mathrm{~h}$.

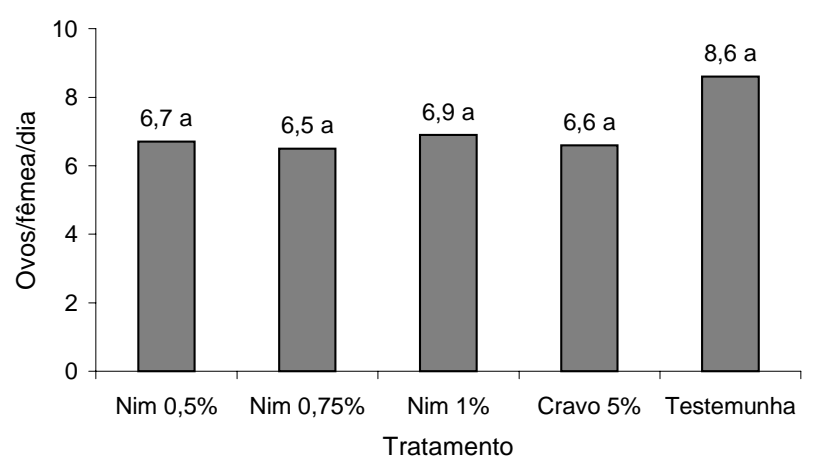

Figura 3 - Oviposição média diária do ácaro verde $M$. tanajoa, em discos de folhas de mandioca tratados com extratos aquosos de nim, A. indica, e cravo-da-índia, $S$. aromaticum. Temp: $27 \pm 0,5^{\circ} \mathrm{C}, 69 \pm 4 \%$ de UR e fotofase de $12 \mathrm{~h}$.

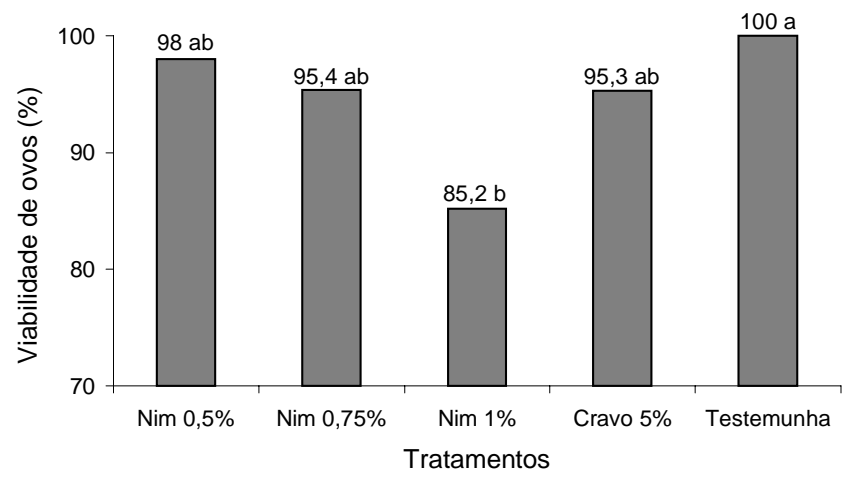

Figura 4 - Viabilidade de ovos do ácaro verde $M$. tanajoa, em discos de folhas de mandioca tratados com extratos aquosos de nim, A. indica, e cravo-da-índia, $S$. aromaticum. Temp: $27 \pm 0,5^{\circ} \mathrm{C}, 69 \pm 4 \%$ de UR e fotofase de $12 \mathrm{~h}$.

TABELA 1 - Duração (média \pm EP) dos estágios imaturos de $M$. tanajoa em discos de folhas de mandioca tratados com extratos aquosos de nim, A. indica, e cravo-da-índia, S. aromaticum. Temp: $27 \pm 0,5^{\circ} \mathrm{C}, 69 \pm 4 \%$ de UR e fotofase de $12 \mathrm{~h}$.

\begin{tabular}{lcccccc}
\hline Estágio & \multicolumn{5}{c}{ Tratamento $^{1}$} & \multirow{2}{*}{ CV (\%) } \\
\cline { 2 - 5 } & Nim 0,5\% & Nim 0,75\% & Nim 1,0\% & Cravo 5,0\% & Testemunha & \\
\hline & $1,1 \pm 0,04 \mathrm{ab}$ & $1,2 \pm 0,01 \mathrm{a}$ & $1,0 \pm 0,25 \mathrm{ab}$ & $1,2 \pm 0,07 \mathrm{a}$ & $0,9 \pm 0,04 \mathrm{~b}$ & 8,0 \\
LV & $(\mathrm{n}=39)$ & $(\mathrm{n}=25)$ & $(\mathrm{n}=11)$ & $(\mathrm{n}=40)$ & $(\mathrm{n}=40)$ & \\
PTC & $0,8 \pm 0,02 \mathrm{a}$ & $0,8 \pm 0,03 \mathrm{a}$ & $0,7 \pm 0,02 \mathrm{a}$ & $0,8 \pm 0,01 \mathrm{a}$ & $0,7 \pm 0,07 \mathrm{a}$ & 16,8 \\
& $(\mathrm{n}=37)$ & $(\mathrm{n}=21)$ & $(\mathrm{n}=10)$ & $(\mathrm{n}=40)$ & $(\mathrm{n}=40)$ & \\
PTN & $0,6 \pm 0,04 \mathrm{a}$ & $0,7 \pm 0,07 \mathrm{a}$ & $0,6 \pm 0,01 \mathrm{a}$ & $0,7 \pm 0,03 \mathrm{a}$ & $0,6 \pm 0,04 \mathrm{a}$ & 18,6 \\
& $(\mathrm{n}=37)$ & $(\mathrm{n}=20)$ & $(\mathrm{n}=10)$ & $(\mathrm{n}=40)$ & $(\mathrm{n}=40)$ & \\
DTC & $0,8 \pm 0,04 \mathrm{~b}$ & $0,9 \pm 0,03 \mathrm{ab}$ & $1,0 \pm 0,25 \mathrm{a}$ & $0,9 \pm 0,03 \mathrm{ab}$ & $0,8 \pm 0,04 \mathrm{~b}$ & 8,3 \\
& $(\mathrm{n}=37)$ & $(\mathrm{n}=19)$ & $(\mathrm{n}=9)$ & $(\mathrm{n}=40)$ & $(\mathrm{n}=40)$ & \\
DTN & $0,9 \pm 0,05 \mathrm{a}$ & $0,9 \pm 0,08 \mathrm{a}$ & $0,8 \pm 0,02 \mathrm{a}$ & $0,8 \pm 0,04 \mathrm{a}$ & $0,9 \pm 0,03 \mathrm{a}$ & 16,3 \\
& $(\mathrm{n}=33)$ & $(\mathrm{n}=18)$ & $(\mathrm{n}=7)$ & $(\mathrm{n}=38)$ & $(\mathrm{n}=40)$ & \\
TLC & $1,0 \pm 0,01 \mathrm{~b}$ & $1,1 \pm 0,04 \mathrm{~b}$ & $1,7 \pm 0,44 \mathrm{a}$ & $1,1 \pm 0,05 \mathrm{~b}$ & $1,1 \pm 0,02 \mathrm{~b}$ & 10,0 \\
& $(\mathrm{n}=32)$ & $(\mathrm{n}=18)$ & $(\mathrm{n}=6)$ & $(\mathrm{n}=38)$ & $(\mathrm{n}=40)$ & \\
\hline
\end{tabular}

${ }^{1}$ Médias seguidas da mesma letra na linha não diferem pelo teste de Tukey ( $\left.\leq 00,05\right)$. (LV) larva; (PTC) protocrisálida; (PTN) protoninfa; (DTC) deutocrisálida; (DTN) deutoninfa; (TLC) teliocrisálida. Temp: $27 \pm 0,5^{\circ} \mathrm{C}, 69 \pm 4 \%$ de UR e fotofase de $12 \mathrm{~h}$. 


\section{AGRADECIMENTOS}

À CAPES e ao CNPq pela concessão das bolsas de estudo e de produtividade em pesquisa, ao primeiro e segundo autores deste trabalho, respectivamente. Ao Engenheiro Agrônomo, José Lamartine Lins Pereira, pelo auxílio na confecção do abstract e ao Prof. Jorge Braz Torres pelas valiosas sugestões.

\section{REFERÊNCIAS BIBLIOGRÁFICAS}

BELLOTTI, A.C.; REYES, J.A.; GUERRERO, J.M.; FERNÁNDEZ, F. Ácaros presentes en el cultivo de la yuca y su control. In: REYES, J.A. (Comp.) Yuca: control integrado de plagas. Cali: PNUD - CIAT, 1983. cap.6, p.283-303.

BELLOTTI, A.C.; SMITH, L.; LAPOINTE, L.S. Recent advances in cassava pest management. Annual Review of Entomology, v.44, p.343-370, 1999.

BYRNE, D.H.; BELLOTTI, A.C.; GUERRERO, J.M. The cassava mites. Tropical Pest Management, v.29, p.378-394, 1983.

BYRNE, D.H.; GUERRERO, J.M.; BELLOTTI, A.C.; GRASEN, V.P.T. Yield and plant growth response of Mononychellus mite resistant and susceptible cassava cultivars under protected vs. Infested conditions. Crop Science, v.22, p.486-490, 1982.

DIMETRY, N.Z.; AMER, S.A.A.; REDA, A.S. Biological activity of two neem seed kernel extracts against the two-spotted spider mite Tetranychus urticae Koch. Journal of Applied Entomology, v.116, p.308-312, 1993.

DORESTE, E.S.; C. ARIAS.; BELLOTTI, A.C. Pruebas de variedades de yuca resistentes a ácaros tetraniquídeos. Revista de la Faculdad de Agronomia, v.10, p.95-104, 1979.

GONÇALVES, M.E. de C.; PEREIRA, F.F.; MAGNO, M.V.; COSTA, A.C.S.; OLIVEIRA, J.V. Efeito de extratos aquosos de plantas sobre o ácaro verde da mandioca Mononychellus tanajoa (Bondar) (Acari: Tetranychidae). In: SIMPÓSIO DE PESQUISA E PÓS-GRADUAÇÃO DA UNIVERSIDADE FEDERAL RURAL DE PERNAMBUCO, 2., Recife, 1999. Resumos. Recife: Universidade Federal Rural de Pernambuco, 1999. p.53.

MANSOUR, F.A.; ASCHER, K.R.S. Effects of neem (Azadirachta indica) seed kernel extract from different solvents, on the carmine spider mite, Tetranychus cinnabarinus. Phytoparasitica, v.11, p.177-185, 1983.

MANSOUR, F.A.; ASCHER, K.R.S.; ABO-MOCH, F. Effects of Margosan- $\mathrm{O}^{\mathrm{TM}}$ and RD9-Repelin ${ }^{\mathrm{R}}$ on spider, and on predacious and phytophagous mites. Phytoparasitica, v.21, p.205211,1993.

MANSOUR, F.A.; ASCHER, K.R.S.; ABO-MOCH, F. Effects of Neemgard on phytophagous and predacious mites and on spiders. Phytoparasitica, v.25, p.333-336, 1997.

MANSOUR, F.A.; ASCHER, K.R.S.; OMARI, N. Effects of neem (Azadirachta indica) seed kernel extract from different solvents, on the predacious mite Phytoseiulus persimilis and the phytophagous mite Tetranychus cinnabarinus. Phytoparasitica, v.15, p.125-130, 1987.
MANSOUR, F.A.; ASCHER, K.R.S.; OMARI, N. Toxicity of neem (Azadirachta indica) seed kernel extract prepared with different solvents, on the spider Chiracanthium mildei. Phytoparasitica, v.14, p.73-76, 1986.

McMURTRY, J.A.; HUFFAKER C.B.; VRIE M.V. Tetranychids enemies: their biological characteres and the impact of spray practices. Hilgardia, v.40, p.331-390, 1970.

MILLER, F.; UETZ, S. Evaluating biorational pesticides for controlling arthropod pests and their phytotoxic effects on greenhouse crops. HortTecnology, v.8, p.185-192, 1998.

MORAES, G.J. Controle biológico de ácaros fitófagos. Informe Agropecuário, v.15, p.55-62, 1991.

NORONHA, A.C.da S.; MORAES, G.J.; SMITH, L. Introdução de ácaros predadores da família Phytoseiidae para o controle biológico do ácaro verde da mandioca Mononychellus tanajoa (Bondar), no Nordeste do Brasil. Cruz das Almas: EMBRAPA, CNPMF, 1995. 3p. (Pesquisa em Andamento, 34).

REMBOLD, H. Azadirachtins, their structure and mode of action. In: ARNASON, J.T.; PHILOGENE, B.J.R.; MORAND, P. (Ed.) Insecticides of plant origin. Washington: ACS, 1989. cap.11, p.150-163.

SAMWAYS, M.J. Imigration, population growth and mortality of insects and mites on cassava in Brazil. Bulletin of Entomological Research, v.69, p.491-505, 1979.

SCHMUTTERER, H. Properties and potential of natural pesticides from the neem tree, Azadirachta indica. Annual Review of Entomology, v.35, p.271-297, 1990.

SPOLLEN, K.M.; ISMAN, M.B. Acute and sublethal effects of a neem insecticide on the commercial biological control agents Phytoseiulus persimilis and Amblyseius cucumeris (Acari: Phytoseiidae) and Aphidoletes aphidimyza (Diptera: Cecidomyiidae). Journal of Economic Entomology, v.89, p.1379-1386, 1996.

SUNDARAM, K.M.S.; CAMPBELL, R.; SLOANE, L.; STUDENS, J. Uptake, translocation, persistence and fate of azadirachtin in aspen plants (Populus tremuloides Michx.) and its effect on pestiferous two-spotted spider mite (Tetranychus urticae Koch). Crop Protection, v.14, p.415-421,1995.

VEIGA, A.F.S.L. Aspectos bioecológicos e alternativas de controle do ácaro verde da mandioca Mononychellus tanajoa (Bondar, 1938) (Acarina:Tetranychidae) no Estado de Pernambuco. Piracicaba, 1985. 137p. Tese (Doutorado) Escola Superior de Agricultura "Luiz de Queiroz", Universidade de São Paulo.

YANINEK, J.S.; HERREN, H.R. Introduction and spread of the cassava green mite, Mononychellus tanajoa (Bondar) (Acari:Tetranychidae), a exotic pest in Africa and the search for appropriate control methods: a review. Bulletin of Entomological Research, v.78, p.1-13, 1988.

YANINEK.; MORAES, G.J.; MARKHAM, R.H. Handbook on the cassava green mite (Mononychellus tanajoa) in África. Ibadan: International Institute of Tropical Agriculture, 1989. 140p.

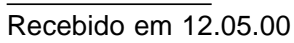

